\title{
Hybrid assembly of the large and highly repetitive genome of Aegilops tauschii, a progenitor of bread wheat, with the MaSuRCA mega-reads algorithm
}

\author{
Aleksey V. Zimin, ${ }^{1,2}$ Daniela Puiu, ${ }^{1}$ Ming-Cheng Luo, ${ }^{3}$ Tingting Zhu, ${ }^{3}$ Sergey Koren, ${ }^{4}$ \\ Guillaume Marçais, ${ }^{2,5}$ James A. Yorke, ${ }^{2,6}$ Jan Dvorák, $^{3}$ and Steven L. Salzberg ${ }^{1,7}$ \\ ${ }^{1}$ Center for Computational Biology, McKusick-Nathans Institute of Genetic Medicine, Johns Hopkins School of Medicine, Baltimore, \\ Maryland 21205, USA; ${ }^{2}$ Institute for Physical Sciences and Technology, University of Maryland, College Park, Maryland 20742, USA; \\ ${ }^{3}$ Department of Plant Sciences, University of California, Davis, California 95616, USA; ${ }^{4}$ National Human Genome Research Institute, \\ National Institutes of Health, Bethesda, Maryland 20892, USA; ${ }^{5}$ Department of Computational Biology, Carnegie Mellon University, \\ Pittsburgh, Pennsylvania 15213, USA; ${ }^{6}$ Departments of Mathematics and Physics, University of Maryland, College Park, Maryland \\ 20742, USA; ${ }^{7}$ Departments of Biomedical Engineering, Computer Science, and Biostatistics, Johns Hopkins University, Baltimore, \\ Maryland 21218, USA
}

\begin{abstract}
Long sequencing reads generated by single-molecule sequencing technology offer the possibility of dramatically improving the contiguity of genome assemblies. The biggest challenge today is that long reads have relatively high error rates, currently around $15 \%$. The high error rates make it difficult to use this data alone, particularly with highly repetitive plant genomes. Errors in the raw data can lead to insertion or deletion errors (indels) in the consensus genome sequence, which in turn create significant problems for downstream analysis; for example, a single indel may shift the reading frame and incorrectly truncate a protein sequence. Here, we describe an algorithm that solves the high error rate problem by combining long, high-error reads with shorter but much more accurate Illumina sequencing reads, whose error rates average $<1 \%$. Our hybrid assembly algorithm combines these two types of reads to construct mega-reads, which are both long and accurate, and then assembles the mega-reads using the $\mathrm{CABOG}$ assembler, which was designed for long reads. We apply this technique to a large data set of Illumina and PacBio sequences from the species Aegilops tauschii, a large and extremely repetitive plant genome that has resisted previous attempts at assembly. We show that the resulting assembled contigs are far larger than in any previous assembly, with an N50 contig size of 486,807 nucleotides. We compare the contigs to independently produced optical maps to evaluate their large-scale accuracy, and to a set of high-quality bacterial artificial chromosome (BAC)-based assemblies to evaluate base-level accuracy.
\end{abstract}

[Supplemental material is available for this article.]

Long-read sequencing technologies have made significant advances in the past few years, with read lengths rapidly increasing while costs steadily dropped. Current technology can yield reads with average lengths of 5-10 kilobases $(\mathrm{kb})$ and a throughput that can reach a gigabase $(\mathrm{Gb})$ from a single Pacific Biosciences (PacBio) SMRT cell. Although this technology remains more expensive and has lower throughput than Illumina sequencing, it is now feasible to generate deep coverage of a large plant or animal genome at a modest cost. The long read lengths are extremely valuable for de novo genome assembly, allowing assemblers to overcome many of the problems caused by repeated sequences that are longer than Illumina reads. This is particularly true for plant genomes, in which transposable elements with lengths $>1 \mathrm{~kb}$ are pervasive, often occupying over half of the genome. In the absence of other linking information, any near-exact repeat longer than a read will create a break in an assembly.

Traditionally, the primary strategy for spanning long repeats has been to create paired-end libraries from long DNA fragments, ranging in size from $2-10 \mathrm{~kb}$, or from even longer fosmids ( 40 $\mathrm{kb})$ or BACs $(\sim 125-150 \mathrm{~kb})$. These strategies yield valuable long-

Corresponding author: salzberg@jhu.edu

Article published online before print. Article, supplemental material, and publication date are at http://www.genome.org/cgi/doi/10.1101/gr.213405.116. range linking information, but they require more complex and more expensive methods of preparing DNA so that both ends can be sequenced. In contrast, when a single read spans a repeat and contains unique flanking sequences, the repeat can be directly incorporated into the assembly without the need to use paired-end information.

Recently, several assembly techniques have been developed for de novo assembly of a large genome from high-coverage (50x or greater) PacBio reads. These include: the PBcR assembler, which employs the MHAP algorithm (Berlin et al. 2015) together with the CABOG assembly system; the HGAP assembler (Chin et al. 2013); the Canu assembler (Koren et al. 2017), which also uses MHAP; and the FALCON assembler developed at Pacific Biosciences (https://github.com/PacificBiosciences/FALCON). Other methods employ a hybrid assembly strategy, in which short Illumina reads are used to correct errors in longer PacBio reads (Koren et al. 2012; Hackl et al. 2014; Salmela and Rivals 2014).

In this paper, we describe a new hybrid assembly technique that can produce highly contiguous assemblies of large genomes

C 2017 Zimin et al. This article is distributed exclusively by Cold Spring Harbor Laboratory Press for the first six months after the full-issue publication date (see http://genome.cshlp.org/site/misc/terms.xhtml). After six months, it is available under a Creative Commons License (Attribution-NonCommercial 4.0 International), as described at http://creativecommons.org/licenses/by-nc/4.0/. 
using a combination of PacBio and Illumina reads. The new method extends Illumina reads into super-reads (Zimin et al. 2013) and then combines these with the PacBio data to create mega-reads, essentially converting each PacBio read into one or more very long, highly accurate reads. The mega-reads software, which is now incorporated into the MaSuRCA assembler, can handle hybrid assemblies of almost any plant or animal genome, including genomes as large as the 22-Gbp loblolly pine. The memory usage of the hybrid assembly algorithm scales linearly with the size of the genome, and its execution time scales linearly with the depth of coverage in PacBio reads. One terabyte of memory is sufficient for most genomes under $10 \mathrm{Gbp}$ in length. Here, we use this method to produce an assembly of the large and complex genome of Aegilops tauschii, one of the three diploid progenitors of bread wheat. The Ae. tauschii genome is unusually repetitive and has proven extremely difficult to assemble using short-read data.

Ae. tauschii is a self-pollinating inbred grass species whose genome is nearly homozygous, making it an excellent asset for evaluation of error rates in assembly. To this end, we have generated an optical BioNano genome (BNG) map for the Ae. tauschii genome, which provided a sequence-independent means of evaluating the large-scale accuracy of the assembly, as we discuss below. We have also independently sequenced the Ae. tauschii genome using an ordered BAC-clone sequencing approach, which provides a means of evaluating the base-level accuracy of the assembly.

\section{Methods}

\section{Sequencing data requirements}

Our hybrid assembly algorithm expects at least $100 \times$ genome coverage by paired Illumina reads of $100-250 \mathrm{bp}$, combined with at least $10 \times$ coverage in PacBio reads. Based on preliminary data, we expect that generating deeper PacBio coverage, up to $60 \times$, is likely to improve the final results. Deeper Illumina coverage may also be beneficial. The mega-reads algorithm has the following main steps.

\section{Super-read construction}

We first transform Illumina paired-end reads into super-reads, as described previously (Zimin et al. 2013). The super-reads algorithm builds a database of all sequences of a user-specified length $k$, and then extends these $k$-mers in both directions as long as the extensions are unambiguous. In most cases, the super-reads will be much longer than the original Illumina reads, typically averaging $400 \mathrm{bp}$ or more, depending on the repetitiveness of the genome. Subsequent steps of our algorithm use the longer but much lower coverage (usually $2-4 \times$ ) super-reads, thus providing a very substantial degree of data compression. Super-reads are longer and have fewer errors than Illumina reads; thus, they can be mapped to high-error PacBio reads more reliably and therefore provide a better vehicle for error correction.

For the next two steps, we treat each PacBio read as a template to which super-reads can be attached, as illustrated in Figure 1.

\section{Approximate alignment along a PacBio read}

We create approximate alignments of the super-reads to each PacBio read using 15-mers that the PacBio read has in common with super-reads. We used 15-mers so that we would have a sufficient number of "seeds" along most PacBio reads; smaller $k$-mers might also work but could increase the spurious match rate. Note that the choice of $k=15$ for this step can be changed for different data sets. We first build a database of all 15-mers in the su-

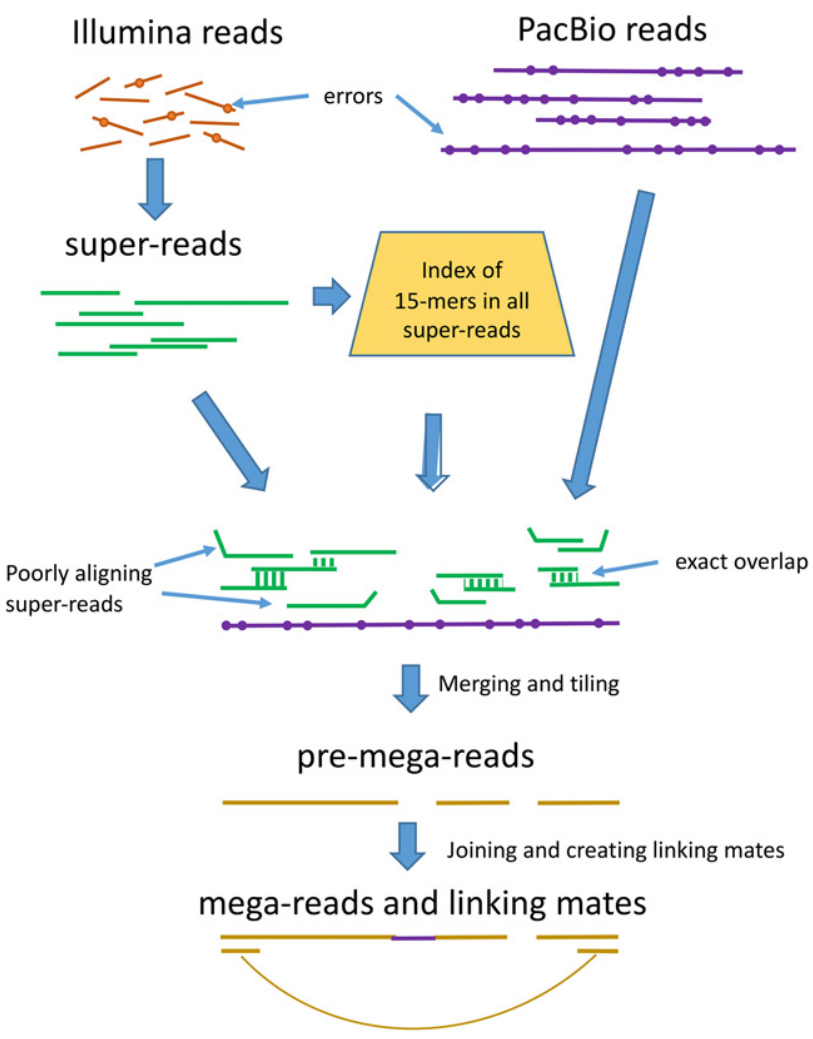

Figure 1. Overview of the mega-reads algorithm. Low-error rate Illumina reads (top left) are used to build longer super-reads (green lines), which in turn are used to construct a database of all 15-mers in those reads. PacBio reads (purple lines) and super-reads are then aligned, using the 15-mer index. Inconsistent super-reads are shown as kinked lines; these are discarded, and the remaining super-reads are merged, using the PacBio read as a template, to produce pre-mega-reads (yellow). These are further merged to produce the final mega-reads and to generate linking mates across gaps.

per-reads and use this database to compute, for each super-read, its approximate start and end positions on each PacBio read. This approach is similar (although different in many details) to both MHAP (Berlin et al. 2015) and minimap (Li 2016), in that both these other algorithms find chains of "seed" alignments in long PacBio reads. Our method does not compute a full alignment.

For each PacBio read $P$, we walk down the read looking at each 15-mer. We use the 15-mer database to determine (in constant time for each 15-mer) which 15-mers are found in super-reads. Once we have the super-reads that match $P$, for each such superread $S$ we look for ordered subsequences of the 15-mers that both $P$ and $S$ have in common. (The 15 -mers can be overlapping.) We then assign a score to each super-read $S$, where the score is the number of 15-mers in the longest common subsequence (LCS) of 15-mers in the two reads. We label an alignment as plausible if the score of $S$ exceeds some specified minimum. For each plausible alignment, we compute an approximate position of $S$ along $P$ based on the positions of the LCS 15-mers in $P$ and $S$.

Note that a super-read can align to many different PacBio reads; the number will depend on the depth of coverage of the PacBio data.

\section{Graph traversal for a PacBio read}

Let $K$ denote the $k$-mer size that was used to generate the superreads. After super-reads are constructed, we record all exact 
overlaps of pairs of super-reads for which the length of the overlap is at least $K$.

Using all super-read positions on a PacBio read $P$, we create possible paths of (plausible) super-reads along $P$. Each path consists of a sequence of super-reads where two adjacent super-reads must have an exact overlap of at least $K$ bases and also must have positions on $P$ that make it possible for them to overlap. We compute an LCS score for each path.

A path might span only part of $P$, and conversely, subsequences of $P$ might not be covered by any path. We then form a graph consisting of the paths along $P$, where super-reads are the nodes and $K$-overlaps are the edges. For each connected component of paths (or more precisely, a connected graph of superreads), we compute the LCS score and we choose the path with the highest score. We call each such path a pre-mega-read. The sequence of a mega-read is essentially a long, high-quality "read" that covers part or all of the original PacBio read $P$.

At this point, each connected component is a directed acyclic graph (DAG) of super-reads that overlap by at least $K$ bases and that align to $P$. The approximate positions of the super-reads on $P$ impose a topological order on the DAG. We impose an overall direction on the DAG from the $5^{\prime}$ end toward the $3^{\prime}$ end of $P$.

\section{Tiling}

We tile the PacBio read $P$ with the pre-mega-reads in a greedy fashion, beginning with the longest pre-mega-read, and disallowing overlaps longer than $K$ bases (Fig. 1). We choose the pre-megareads for $P$ by maximizing the total of all LCS scores in the tiling.

Many PacBio reads will be tiled by more than one pre-megaread; i.e., the tiling has gaps. Gaps might be caused by lack of Illumina read coverage for parts of the genome, or by long stretches of poor-quality sequence in a PacBio read, or (rarely) by chimeric PacBio reads. Even though we have PacBio sequence spanning these gaps, we choose not to simply merge the premega-reads using raw PacBio read sequence because that might create stretches of low-quality sequence in the mega-reads. However, if multiple PacBio reads overlap one another for the sequence in one of these gaps, we can sometimes fill the gap between pre-mega-reads. We only use raw PacBio read sequence to join neighboring pre-mega-reads if (1) the tilings for at least three PacBio reads have nearly identical gaps, (2) the pre-mega-reads surrounding the gap have identical sequence, and (3) the gap lengths are nearly identical. Here, gap length is determined by the length of the PacBio sequence between the aligned premega-reads. If these conditions are met, we compute the gap-filling sequence from the original PacBio reads that span the gap. The consensus step in the CABOG assembler then creates the final version of the sequence. For the Ae. tauschii assembly, the average length of filled-in gaps was $206 \mathrm{bp}$, and the final assembly contained a total of $14 \mathrm{Mbp}(0.03 \%)$ that resulted from filling gaps, of which $5.8 \mathrm{Mb}(0.014 \%)$ was covered by the minimum of three PacBio reads.

It is also possible that a gap in the tiling is not a gap at all but instead is an erroneous insertion in the PacBio read. In these cases, the pre-mega-reads flanking the gap may overlap one another. If the pre-mega-reads overlap by at least $37 \mathrm{bp}$, then we merge them to close the gap.

Note that the user can set the maximum gap size for the gapfilling procedure, and the algorithm will not attempt to fill gaps larger than this maximum. If one sets the maximum gap size to zero, the mega-reads assembler will not use raw PacBio sequence at all and will only join pre-mega-reads when they have an exact overlap of 37 bases or more. For Ae. tauschii, the maximum gap size was $750 \mathrm{bp}$.
The result of this tiling and gap-filling process is the final set of mega-reads.

\section{Creating linking pairs}

When mega-reads cannot be merged and a gap remains, we create a linked pair of "reads" that spans the gap. We extract two 500-bp sequences from the mega-reads flanking the gap and link them together as mates (Fig. 1). (If either mega-read is $<500 \mathrm{bp}$, we create a shorter linking read.) The assembler uses these sequences in its scaffolding step to ensure that all mega-reads from the same PacBio read are kept adjacent in the assembly; i.e., they are placed into the same scaffold. We call these artificial mates the linking pairs.

\section{Assembly}

Finally, we assemble the mega-reads along with the linking pairs into contigs and scaffolds using the CABOG assembler (Miller et al. 2008). For this step, we can also use other linking information, if available, for scaffolding.

\section{Results}

\section{Data sets}

We generated over 19 million PacBio reads, equivalent to $\sim 38 \times$ genome coverage, using the SMRT P6-C4 chemistry. We also generated a total of $177 \times$ coverage on an Illumina HiSeq 2500 in paired 200-bp reads and an Illumina MiSeq with paired 250-bp reads (Table 1). These data sets were the only input used for our hybrid assembly of Ae. tauschii.

\section{Ae. tauschii assembly}

The methods described above produced 16.7 M super-reads (using a $k$-mer length of 127) from the Illumina data, and 18.7 M megareads from the super-reads and PacBio reads (Table 2). We used the CABOG assembler (version wgs-8.3rc2) in the MaSuRCA mega-reads pipeline to produce an initial assembly of the megareads. This assembly contained 128,898 scaffolds totaling 4.778 $\mathrm{Gb}$ in length. We then manually ran a post-processing step that included four rounds of aligning each scaffold to all others, in order to remove scaffolds that were either duplicated or that were completely contained within other scaffolds. For this alignment step, we ran BWA-MEM (Li 2013) with parameters -k127 -e and then used NUCmer (Delcher et al. 2002) to find and remove duplicate alignments. This procedure identified a total of 75,338 scaffolds (most of them very small) that were contained in other scaffolds and could be safely removed. Total computational time for all steps of the assembly was approximately 110,000 CPU hours, with about 72,000 CPU hours for computing super-reads

Table 1. Input data used for the Ae. tauschii hybrid assembly

\begin{tabular}{lccc}
\hline Sequence data type & $\begin{array}{c}\text { Number of } \\
\text { reads }\end{array}$ & $\begin{array}{c}\text { Average } \\
\text { read length } \\
\text { (bp) }\end{array}$ & $\begin{array}{c}\text { Genome } \\
\text { coverage }\end{array}$ \\
\hline Illumina HiSeq paired-end & $1.98 \times 10^{9}$ & 200 & $93.2 \times$ \\
Illumina MiSeq paired-end & $1.41 \times 10^{9}$ & 250 & $83.6 \times$ \\
PacBio SMRT P6-C4 & $1.92 \times 10^{7}$ & 8519 & $38.5 \times$ \\
\hline
\end{tabular}

Coverage is computed based on an estimated genome size of $4.25 \mathrm{~Gb}$. Paired Illumina reads were generated from fragments whose lengths averaged $450-500 \mathrm{bp}$. 
Table 2. Statistics for super-reads and mega-reads

\begin{tabular}{lcccrc}
\hline & Number & Coverage & $\begin{array}{c}\text { Average } \\
\text { length (bp) }\end{array}$ & $\begin{array}{r}\text { N50 } \\
\text { (bp) }\end{array}$ & $\begin{array}{r}\text { Error } \\
\text { rate } \\
\text { (\%) }\end{array}$ \\
\hline Super-reads & $16.7 \times 10^{6}$ & $1.9 \times$ & 474 & 749 & $<0.09$ \\
Mega-reads & $18.7 \times 10^{6}$ & $27.8 \times$ & 6319 & 9378 & $<0.23$ \\
\hline
\end{tabular}

Super-reads were constructed from Illumina data, and mega-reads were constructed as described in the main text. Coverage is based on an estimated genome size of $4.25 \mathrm{Gbp}$. Error rates were computed by mapping the reads against Illumina-only contigs.

and mega-reads, and the remaining time spent in assembly of contigs and scaffolds. The code is highly parallelized so that most procedures were run in parallel on large computing grids.

The resulting Ae. tauschii assembly, version Aet_MR.1.0, contains 53,560 scaffolds with a total span of $4.338 \mathrm{~Gb}$, a contig N50 size of 486,807 bp, and a scaffold N50 size of 521,653 bp (Table 3). As described above, scaffolding was minimal because it used only the linking pairs created from mega-reads that flanked gaps in the original PacBio reads. Thus, for every gap internal to a scaffold, we have at least one PacBio read spanning the gap. The principal benefit of PacBio reads and of the mega-reads algorithm is the much larger contigs that result, $\sim 30$ times larger than the contigs from an Illumina-only assembly (Table 3 ).

We also created a whole-genome assembly with the PacBio reads only, using the Canu assembler (Berlin et al. 2015). For this assembly, we generated additional data to bring the PacBio coverage up to $55 x$. The Canu assembly had a total length of $4.06 \mathrm{~Gb}$ and an N50 size (using $4.25 \mathrm{~Gb}$ as the genome size) of 311,860 bp (Table 3). It is worth noting here that the Aet_MR.1.0 assembly has many more contigs than the Canu assembly, but this is due to a large number of small contigs in the tail of the distribution and to the fact that the Aet_MR.1.0 assembly is $\sim 264 \mathrm{Mb}$ larger. If we select contigs from the Aet_MR.1.0 assembly whose sizes total 4.06 $\mathrm{Gb}$ (the same total as the Canu assembly), we need only 24,309 contigs, almost exactly the same number as in the Canu assembly, and the smallest such contig is $15,911 \mathrm{bp}$. The total computation time for the Canu assembly was 38,000 CPU hours, while the mega-reads assembly took 110,000 hours. However, expected improvements in these algorithms suggest that the two assemblers would take less computational time today and will likely continue to improve.

\section{Evaluation of assembly quality}

We evaluated the quality of the output contigs using two metrics: large-scale contiguity and consensus sequence accuracy. To evaluate large-scale accuracy, we used an independently constructed BioNano Genomics (BNG) map. This technology, developed by BioNano Genomics, allows the construction of accurate maps based on restriction enzymes, in which DNA molecules are passed through a nanochannel and fluorescently tagged restriction sites are detected (Lam et al. 2012). This process creates many small restriction-mapped regions that can span several megabases each. BNG maps have recently been used to improve the assembly of portions of several highly repetitive plant genomes, including one arm of the bread wheat genome (Stankova et al. 2016), a 2$\mathrm{Mb}$ fragment of Ae. tauschii (Hastie et al. 2013), and six small but complex regions of the maize genome (Dong et al. 2016). To use these maps to assess quality of a sequence assembly, the distances and positions of the same restriction sites along the BNG map are compared with a restriction map constructed computationally.

Here, we used the Ae. tauschii BNG map only to evaluate the correctness of Aet_MR.1.0; it was not used to construct or modify the assembly. We aligned our contigs to the BNG map using the restriction sites in the contigs, and searched for regions of disagreement, which indicate either an error in the BNG map or a misassembled contig. We only considered alignments that shared at least 15 restriction sites, which in most cases meant the aligned contigs were longer than $140 \mathrm{~kb}$. Note that the BNG map does not contain sequence, and the only errors this procedure can detect are relatively large-scale rearrangements, insertions, or deletions.

We found 572 locations where a contig disagreed with the BNG map. All disagreements were apparent chimeric joins in the contigs with respect to the BNG map. A typical signature of a misassembly is a sharp drop in coverage, indicating a possible "weak" overlap holding the contig together. Given that the average coverage in mega-reads was $\sim 28 \times$ (Table 2 ), we flagged as a possible misassembly any disagreement with the BNG map where the coverage was $<4$; this analysis flagged 342 locations. We examined a small set of the higher-coverage discrepancies manually, and in each case the assembly appeared correct (based on underlying support from paired Illumina reads); thus, we concluded that these are likely to represent errors in the BNG map rather than in Aet_MR.1.0. Therefore, for the full assembly of $4.28 \mathrm{~Gb}$, we estimate approximately one assembly error per $12.5 \mathrm{Mb}$.

To estimate base-level accuracy, we aligned a subset of the PacBio reads (50,000 randomly sampled reads), the super-reads, the mega-reads, and the final set of assembled contigs to the previously released Ae. tauschii assembly, version 0.4 (ftp://ftp.ccb.jhu. edu/pub/data/Aegilops_tauschii). That assembly was built from 5216 pools of bacterial artificial chromosomes (BACs), with each pool containing eight overlapping BACs spanning approximately $1 \mathrm{Mb}$. (Each BAC was a haploid sequence averaging $\sim 120 \mathrm{~kb}$ in length.) These pools were independently assembled from 250-bp Illumina MiSeq reads using SOAPdenovo2 (Luo et al. 2012). The reads used for the BAC pool assemblies were a subset of reads used for creating the mega-reads. For alignment, we used the NUCmer program from the MUMmer package (Delcher et al. 2002; Kurtz et al. 2004) with a minimum match length of 127 bp to anchor each alignment.

The average identity of the alignments was $85.1 \%$, 99.91\%, and $99.77 \%$ for the PacBio reads, super-reads, and mega-reads, respectively. From this, we estimate that the error rates for super-

Table 3. Assembly statistics for Ae. tauschii Aet_MR.1.0 compared to other assemblies

\begin{tabular}{lllc}
\hline Assembler & \multicolumn{1}{c}{$\begin{array}{c}\text { MaSuRCA } \\
\text { mega-reads }\end{array}$} & \multicolumn{1}{c}{ Canu } & SOAPdenovo $^{\mathbf{a}}$ \\
\hline $\begin{array}{l}\text { Total assembly } \\
\text { length (bp) }\end{array}$ & $4,328,138,807$ & $4,064,667,949$ & $2,691,663,445$ \\
$\begin{array}{l}\text { N50 contig size (bp) } \\
\text { Number of contigs }\end{array}$ & 486,807 & 311,860 & 2105 \\
\hline
\end{tabular}

N50 numbers were computed using an estimated genome size of 4.25 $\mathrm{Gb}$. The Canu assembly used PacBio data only but at deeper coverage, $55 x$. The SOAPdenovo ( $\mathrm{Li}$ et al. 2010) assembly reported in jia et al. (2013) used 94x coverage in Illumina and Roche 454 sequences. The MaSuRCA mega-reads assembly used only the short-fragment Illumina pairs plus the PacBio data.

alia et al. 2013.

\section{Genome Research}

www.genome.org 
reads and mega-reads were $<0.09 \%$ and $0.23 \%$, respectively (Table $2)$. The mega-reads have a higher error rate because some of them include small patches of sequence computed from alignments of PacBio-only data. The PacBio error rate of $14.9 \%$ was consistent with previous reports on this technology.

For the final assembly, we computed the average identity for all alignments longer than $10 \mathrm{~kb}$, which is long enough to span almost all repeats in the genome. This yielded 10,597 alignments covering 1,629,700,561 bp, with an average identity of $99.96 \%$ (one mismatch every 2500 bases), with values ranging from $99.24 \%$ to $100 \%$. Thus, the base-level accuracy of the assembly appears very high: note that some differences between the haploid BAC assemblies and the diploid whole-genome assembly are likely due to haplotype differences rather than errors. In support of this hypothesis, if we consider only the alignments at $99.99 \%$ identity, which presumably come from regions of the whole-genome assembly whose haplotype matches the BAC sequence, these cover $650,572,225$ bp (40\%) of the aligned regions. Note that we also evaluated the base-level accuracy of the Canu assembly using the same procedure, which yielded a rate of $99.32 \%$ identity between the contigs and the BAC assemblies.

\section{Assembly quality as a function of sequencing depth}

To evaluate how the mega-reads assembly method performs with varying amounts of PacBio data, we performed a series of experiments on a data set from Arabidopsis thaliana Ler-0. We used a hybrid data set for this genome generated previously (Lee et al. 2014), which includes over $100 \times$ coverage by PacBio reads using P5-C4 chemistry and $110 \times$ coverage by paired, $300 \mathrm{bp}$ Illumina MiSeq reads. We sampled the PacBio data to create six data sets ranging from $10 \times$ to $60 \times$ coverage and combined each of these with $100 \times$ coverage in MiSeq reads. Figure 2 shows how the resulting assembly N50 size varies with PacBio coverage. The N50 size was greatest at a depth of $60 \times$. Note that because this genome is far less repetitive than Ae. tauschii, the assembly was much more contiguous; also note that with newer P6-C4 chemistry, which produces longer reads, the results with lower PacBio coverage would very likely improve.

\section{Discussion}

Both Ae. tauschii and its close relative, hexaploid wheat (Triticum aestivum), have proven difficult to assemble because of their unusually high proportion of repetitive sequences. A previously published version of Ae. tauschii (Jia et al. 2013) yielded only $2.69 \mathrm{Gbp}$ ( $\sim 63 \%$ of the genome) spread across 1.1 million contigs. Attempts to assemble $T$. aestivum have met with similar problems: a massive effort to sequence T. aestivum chromosome-by-chromosome yielded only $61 \%$ of the genome in very small contigs with N50 sizes from 1.7 to $8.9 \mathrm{~kb}$ (International Wheat Genome Sequencing 2014). Most of the repeats in Ae. tauschii and in other plants consist of transposons (Lisch 2013), which occur in thousands of copies, many of them nearly identical, throughout the genome. Assembly algorithms can find the correct location for these elements if the input data include reads that are long enough to contain the entire span of a repeat plus unique flanking regions on either side.

The PacBio reads generated in this study, with an average read length of $8520 \mathrm{bp}$, are easily long enough to span most transposable elements, which are usually $2-3 \mathrm{~kb}$ in length (though some can be longer). However, the high error rate of PacBio reads requires some form of error correction before these sequences can be used in a final assembly. The mega-reads introduced here solve both these problems: with an average length of $6319 \mathrm{bp}$, they are long enough to contain the ubiquitous $2-3 \mathrm{~kb}$ repeats in the $\mathrm{Ae}$. tauschii genome, and they are accurate enough-much more accurate than raw Illumina reads-to be used to generate a high-quality assembly. Using these mega-reads, we have generated a whole-genome assembly of Ae. tauschii with an N50 contig size of 486,807 $\mathrm{bp}$, more than 20 times longer than any previous assembly. The unprecedented contiguity of this assembly provides a strong foundation for additional mapping and assembly work to create a far more complete picture of this important plant genome. The strategy described here, using deep coverage Illumina sequencing with moderate coverage PacBio sequencing, demonstrates a cost-effective approach to generating highly contiguous, accurate assemblies of large genomes, even when those genomes contain large numbers of long, near-identical repeats.

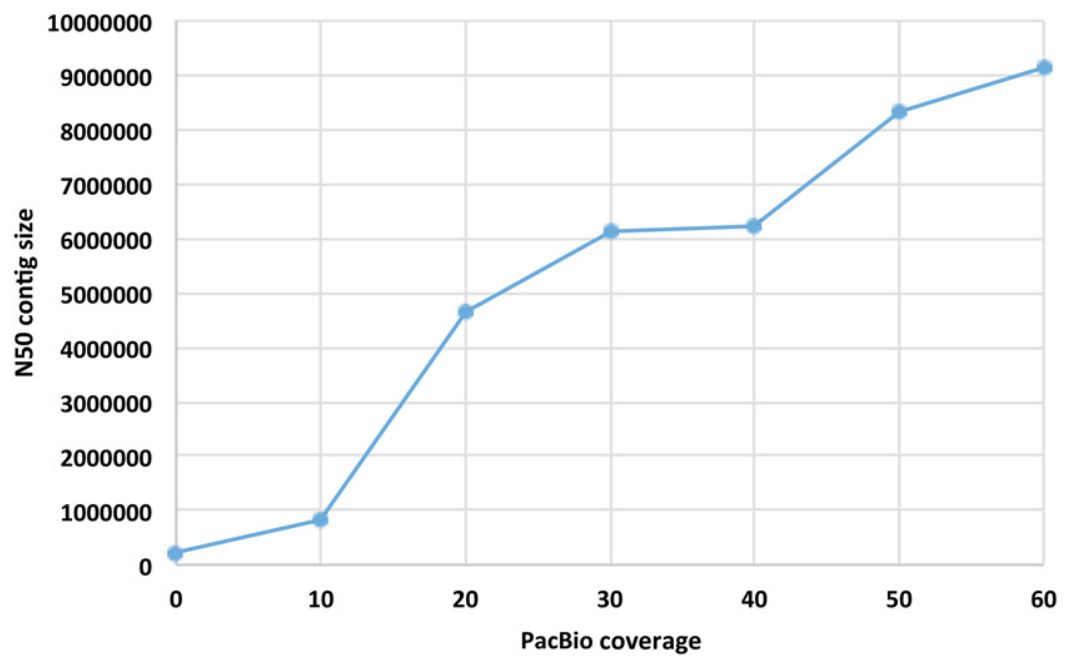

Figure 2. Change in the N50 contig size of genome assemblies using the mega-reads algorithm with varying PacBio coverage and 100x Illumina coverage for the Arabidopsis thaliana genome. At $60 \times$, the N50 size of $9.15 \mathrm{Mb}$ approaches the maximum possible N50 contig size for this genome, which is determined by the sizes of the chromosome arms.

\section{Software availability}

The MaSuRCA mega-reads software is freely available from http://genome. umd.edu/masurca.html and as Supplementary Material.

\section{Data access}

The Ae. tauschii assembly (Aet_MR.1.0) as well as the Illumina and PacBio sequencing data from this study have been submitted to NCBI BioProject (https://www .ncbi.nlm.nih.gov/bioproject/) under accession number PRJNA329335.

\section{Acknowledgments}

This work was supported in part by National Science Foundation (NSF) grant IOS-1238231 to J.D. and by National Institutes of Health (NIH) grant R01 
HG006677 to S.L.S. S.K. was supported by the Intramural Research Program of the National Human Genome Research Institute, NIH. This study utilized the computational resources of the Biowulf system (http://biowulf.nih.gov) at NIH and the MARCC system (http ://marcc.jhu.edu) at JHU.

\section{References}

Berlin K, Koren S, Chin CS, Drake JP, Landolin JM, Phillippy AM. 2015 Assembling large genomes with single-molecule sequencing and locality-sensitive hashing. Nat Biotechnol 33: 623-630.

Chin CS, Alexander DH, Marks P, Klammer AA, Drake J, Heiner C, Clum A, Copeland A, Huddleston J, Eichler EE, et al. 2013. Nonhybrid, finished microbial genome assemblies from long-read SMRT sequencing data. Nat Methods 10: 563-569.

Delcher AL, Phillippy A, Carlton J, Salzberg SL. 2002. Fast algorithms for large-scale genome alignment and comparison. Nucleic Acids Res 30 2478-2483.

Dong J, Feng Y, Kumar D, Zhang W, Zhu T, Luo MC, Messing J. 2016. Analysis of tandem gene copies in maize chromosomal regions reconstructed from long sequence reads. Proc Natl Acad Sci 113: 7949-7956.

Hackl T, Hedrich R, Schultz J, Forster F. 2014. proovread: large-scale high-accuracy PacBio correction through iterative short read consensus. Bioinformatics 30: 3004-3011.

Hastie AR, Dong L, Smith A, Finklestein J, Lam ET, Huo N, Cao H, Kwok PY, Deal KR, Dvorak J, et al. 2013. Rapid genome mapping in nanochannel arrays for highly complete and accurate de novo sequence assembly of the complex Aegilops tauschii genome. PLoS One 8: e55864.

International Wheat Genome Sequencing Consortium. 2014. A chromosome-based draft sequence of the hexaploid bread wheat (Triticum aestivum) genome. Science 345: 1251788.

Jia J, Zhao S, Kong X, Li Y, Zhao G, He W, Appels R, Pfeifer M, Tao Y, Zhang $\mathrm{X}$, et al. 2013. Aegilops tauschii draft genome sequence reveals a gene repertoire for wheat adaptation. Nature 496: 91-95.

Koren S, Schatz MC, Walenz BP, Martin J, Howard JT, Ganapathy G, Wang Z, Rasko DA, McCombie WR, Jarvis ED, et al. 2012. Hybrid error correction and de novo assembly of single-molecule sequencing reads. Nat Biotechnol 30: 693-700.
Koren S, Walenz BP, Berlin K, Miller JR, Bergman NH, Phillippy AM. 2017 Canu: scalable and accurate long-read assembly via adaptive $k$-mer weighting and repeat separation. Genome Res (this issue). doi: 10.1101/gr.215087.116.

Kurtz S, Phillippy A, Delcher AL, Smoot M, Shumway M, Antonescu C, Salzberg SL. 2004. Versatile and open software for comparing large genomes. Genome Biol 5: R12.

Lam ET, Hastie A, Lin C, Ehrlich D, Das SK, Austin MD, Deshpande P, Cao H, Nagarajan N, Xiao M, et al. 2012. Genome mapping on nanochannel arrays for structural variation analysis and sequence assembly. Nat Biotechnol 30: 771-776.

Lee H, Gurtowski J, Yoo S, Marcus S, McCombie WR, Schatz M. 2014. Error correction and assembly complexity of single molecule sequencing reads. bioRxiv doi: 10.1101/006395.

Li H. 2013. Aligning sequence reads, clone sequences and assembly contigs with BWA-MEM. arXiv:1303.3997v1.

Li H. 2016. Minimap and miniasm: fast mapping and de novo assembly for noisy long sequences. Bioinformatics 32: 2103-2110.

Li R, Zhu H, Ruan J, Qian W, Fang X, Shi Z, Li Y, Li S, Shan G, Kristiansen K, et al. 2010. De novo assembly of human genomes with massively parallel short read sequencing. Genome Res 20: 265-272.

Lisch D. 2013. How important are transposons for plant evolution? Nat Rev Genet 14: 49-61.

Luo R, Liu B, Xie Y, Li Z, Huang W, Yuan J, He G, Chen Y, Pan Q, Liu Y, et al. 2012. SOAPdenovo2: an empirically improved memory-efficient shortread de novo assembler. Gigascience 1: 18.

Miller JR, Delcher AL, Koren S, Venter E, Walenz BP, Brownley A, Johnson J, Li K, Mobarry C, Sutton G. 2008. Aggressive assembly of pyrosequencing reads with mates. Bioinformatics 24: $2818-2824$.

Salmela L, Rivals E. 2014. LoRDEC: accurate and efficient long read error correction. Bioinformatics 30: 3506-3514.

Stankova H, Hastie AR, Chan S, Vrana J, Tulpova Z, Kubalakova M, Visend $\mathrm{P}$, Hayashi S, Luo M, Batley J, et al. 2016. BioNano genome mapping of individual chromosomes supports physical mapping and sequence assembly in complex plant genomes. Plant Biotechnol J 14: 1523-1531.

Zimin AV, Marcais G, Puiu D, Roberts M, Salzberg SL, Yorke JA. 2013. The MaSuRCA genome assembler. Bioinformatics 29: 2669-2677.

Received July 26, 2016; accepted in revised form January 18, 2017.

\section{Genome Research}

www.genome.org 


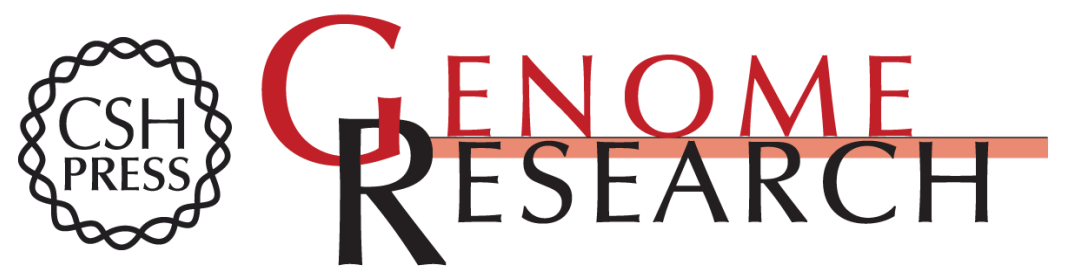

\section{Hybrid assembly of the large and highly repetitive genome of Aegilops tauschii, a progenitor of bread wheat, with the MaSuRCA mega-reads algorithm}

Aleksey V. Zimin, Daniela Puiu, Ming-Cheng Luo, et al.

Genome Res. 2017 27: 787-792 originally published online January 27, 2017

Access the most recent version at doi:10.1101/gr.213405.116

Supplemental
Material http://genome.cshlp.org/content/suppl/2017/04/06/gr.213405.116.DC1

References This article cites 21 articles, 5 of which can be accessed free at:

http://genome.cshlp.org/content/27/5/787.full.html\#ref-list-1

Creative This article is distributed exclusively by Cold Spring Harbor Laboratory Press for the Commons first six months after the full-issue publication date (see

License http://genome.cshlp.org/site/misc/terms.xhtml). After six months, it is available under a Creative Commons License (Attribution-NonCommercial 4.0 International), as described at http://creativecommons.org/licenses/by-nc/4.0/.

Email Alerting Receive free email alerts when new articles cite this article - sign up in the box at the Service top right corner of the article or click here.

\section{Affordable, Accurate Sequencing.}

To subscribe to Genome Research go to:

https://genome.cshlp.org/subscriptions 